\title{
A supersymmetric Skyrme model
}

\author{
Sven Bjarke Gudnason, ${ }^{a}$ Muneto Nitta ${ }^{b}$ and Shin Sasaki ${ }^{c}$ \\ ${ }^{a}$ Institute of Modern Physics, Chinese Academy of Sciences, \\ Lanzhou 730000, China \\ ${ }^{b}$ Department of Physics, and Research and Education Center for Natural Sciences, \\ Keio University, \\ Hiyoshi 4-1-1, Yokohama, Kanagawa 223-8521, Japan \\ ${ }^{c}$ Department of Physics, Kitasato University, \\ Sagamihara 252-0373, Japan \\ E-mail: bjarke@impcas.ac.cn, nitta@phys-h.keio.ac.jp, \\ shin-s@kitasato-u.ac.jp
}

ABSTRaCT: Construction of a supersymmetric extension of the Skyrme term was a longstanding problem because of the auxiliary field problem; that is, the auxiliary field may propagate and cannot be eliminated, and the problem of having fourth-order time derivative terms. In this paper, we construct for the first time a supersymmetric extension of the Skyrme term in four spacetime dimensions, in the manifestly supersymmetric superfield formalism that does not suffer from the auxiliary field problem. Chiral symmetry breaking in supersymmetric theories results not only in Nambu-Goldstone (NG) bosons (pions) but also in the same number of quasi-NG bosons so that the low-energy theory is described by an $\mathrm{SL}(N, \mathbb{C})$-valued matrix field instead of $\mathrm{SU}(N)$ for $\mathrm{NG}$ bosons. The solution of auxiliary fields is trivial on the canonical branch of the auxiliary field equation, in which case our model results in a fourth-order derivative term that is not the Skyrme term. For the case of $\mathrm{SL}(2, \mathbb{C})$, we find explicitly a nontrivial solution to the algebraic auxiliary field equations that we call a non-canonical branch, which when substituted back into the Lagrangian gives a Skyrme-like model. If we restrict to a submanifold, where quasi-NG bosons are turned off, which is tantamount to restricting the Skyrme field to $\mathrm{SU}(2)$, then the fourthorder derivative term reduces exactly to the standard Skyrme term. Our model is the first example of a nontrivial auxiliary field solution in a multi-component model.

Keywords: Supersymmetric Effective Theories, Solitons Monopoles and Instantons

ARXIV EPRINT: 1512.07557 


\section{Contents}

1 Introduction 1

2 The formalism 4

2.1 General action 4

2.2 Chiral symmetry breaking 6

3 The supersymmetric Skyrme term $\quad 7$

3.1 A fourth-order derivative term in the chiral Lagrangian 8

$\begin{array}{lll}3.2 & \text { Canonical branch } & 8\end{array}$

3.3 Non-canonical branch: a supersymmetric Skyrme term 9

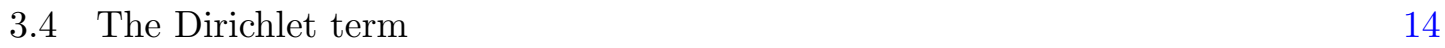

4 Gauging the global symmetry $\quad 15$

$\begin{array}{llr}5 & \text { Conclusion and discussion } & 16\end{array}$

\section{Introduction}

The Skyrme model was first introduced as a toy model describing baryons in a low-energy mesonic field theory $[1,2]$. Later it was shown to be the low-energy limit of large- $N_{c}$ QCD [3, 4]. After this it gained popularity as a model of nuclei in the literature. It took, however, some time before the numerical calculations (and the computing power) could tackle solutions for higher baryon numbers. The breakthrough came with the introduction of the rational maps as an approximation to the real Skyrmion solution $[5,6]$. These are very useful as initial guesses for numerical calculations. For vanishing pion mass, the fullerenes adequately described by the rational maps are believed to be the global minimizers of the Skyrmion energy functional. Once a pion mass of the order of the physical pion mass is introduced, the Skyrmions prefer to order themselves in a lattice of $B=4$ cubes, which can be interpreted as a crystal of alpha particles [7].

Quite a few phenomenologically appealing results have been achieved in the Skyrme model; for recent works, see e.g. [8-11]. A withstanding problem of the Skyrme model, is that the binding energies naturally come out too large (by about an order of magnitude). For this reason, quite some work has been devoted to finding a BPS limit of the Skyrme model. The minimal (original) Skyrme model has a BPS bound [12], that, however, can be saturated only on the 3-sphere [13]. Recently, a different model has been suggested, called the BPS Skyrme model [14, 15], which has a BPS limit and many exact solutions have been found [16]. Naively, one may think that the BPS limits of the Skyrme model above are related to supersymmetry, as is the case for Abrikosov-Nielsen-Olesen vortices or for 't Hooft-Polyakov monopoles [17]. This is, however, not the case for the Skyrme model. 
There have been several attempts in the literature to construct supersymmetric extensions of the Skyrme model, but all attempts failed and it turns out that this is indeed a very difficult problem. The first attempt was made already three decades ago by BergshoeffNepomechie-Schnitzer [18]. The monkey-wrench is that the target space must be Kähler in order for a supersymmetrization to be possible [19]. $S^{3}$, the target space of the Skyrme model, is not Kähler (and neither is $S^{4}$ ). Ref. [18] chose to work around that obstacle by gauging a $\mathrm{U}(1)$ subgroup of the right-acting $\mathrm{SU}(2)$ group. This gauging reduces the target space to $\mathbb{C} P^{1}$, which is the poster boy for Kähler spaces. Their supersymmetrized model has, however, a typical feature of supersymmetric higher-derivative models, namely that it possesses a term with four time derivatives. Or in other words, the Skyrme term is accompanied with an unwanted fourth-order time derivative term. This in turn makes a Hamiltonian formulation impossible. Essentially the same model was obtained by Freyhult [20] in an attempt to supersymmetrize the Faddeev-Skyrme model for Hopfions [21]. More recently, one proposal was made in ref. [22], but it is not supersymmetric because a non-supersymmetric constraint $\left|\phi_{1}\right|^{2}+\left|\phi_{2}\right|^{2}=1$, was put by hand for the chiral superfields $\phi_{1,2} \cdot{ }^{1}$

A more notorious problem called the auxiliary field problem arises when considering higher-derivative models with manifest supersymmetry. The problem is that once derivatives act on the auxiliary field, its equation of motion becomes dynamical instead of algebraic. This means that the auxiliary field becomes propagating and cannot simply be eliminated. This problem is related to the above mentioned problem and in fact was encountered in both ref. [18] and [20]. Two situations occur. If the derivatives act on the auxiliary field $F$ as $X \partial F$, then the problem can be avoided by adding a total derivative of the form $-\partial(X F)$ giving a term $-F \partial X$ in the action and hence a $-\partial X$ in the algebraic equation of motion for $F$. The second case is the troublemaker. If a term like $\partial F \partial F$ occurs, then a simple total derivative added to the action cannot mend the problem. If the second type of terms does not appear, then a supersymmetric theory without the auxiliary field problem can be constructed. First examples of such constructions include refs. [24-29]. The manifestly supersymmetric term found in ref. [25, 26] offers a manifestly supersymmetric class of higher-derivative theories - free from the auxiliary field problem — and has been further studied recently in refs. [30-36]. As is clear from the above discussion, one off-shell supersymmetric Lagrangian gives two (or more) possibilities for Lagrangians in terms of component fields. The first possibility is to set the auxiliary field to zero $(F=0)$, which is possible if no superpotential is turned on; we call this the canonical branch. The other possibility is to find a nontrivial solution for $F$ from its equation of motion, which is algebraic because our construction avoids the auxiliary field problem. We call this the non-canonical branch.

This non-canonical branch is in fact key to constructing a supersymmetric extension of the Skyrme term. It was found in ref. [33] (see also refs. [34, 36, 37]) that this was the case for the baby Skyrme model, that is a $2+1$ dimensional analog of the Skyrme model. Namely, in this case, a supersymmetric baby Skyrme term free from the auxiliary field

\footnotetext{
${ }^{1}$ The model may be made supersymmetric by the introduction of a vector multiplet, which would reduce the target space to $\mathbb{C} P^{1}$, similarly to Bergshoeff-Nepomechie-Schnitzer's approach, but for a sixth-order derivative term [23]. The model is thus a $\mathbb{C} P^{1}$-type model and the Skyrme model is not in its subspace.
} 
problem and four time derivatives was constructed, although the ordinary kinetic term cannot be included. A BPS baby Skyrmion is of a compacton type [33] and is a $1 / 4$ BPS state $[34,36]$ preserving a quarter of the original supersymmetry.

Higher derivatives usually come about in low-energy effective theories by means of integrating out heavy states/fields. Often in the low-energy limit of a theory, some global symmetries are broken spontaneously by the vacuum. In this case the low-energy effective theory is described by the Nambu-Goldstone (NG) bosons of the symmetry breaking, dictated by the symmetries of the vacuum. More precisely, the dynamics of the NG bosons corresponding to a spontaneous symmetry breaking from $G$ to $H$, is described by a nonlinear sigma model whose target space is given by the coset $G / H[38,39]$. A prime (and most famous) example is chiral symmetry breaking in $\mathrm{QCD}$, giving rise to the three pions.

When working with a supersymmetric theory, spontaneous symmetry breaking of global symmetries can of course still take place. The situation is, however, modified with respect the standard case which we just mentioned above [40-42]. Spontaneous symmetry breaking in a supersymmetric theory is tantamount to a chiral superfield acquiring a vacuum expectation value (VEV) due to the superpotential $W$ (F-term condition). The superpotential is a holomorphic functional and hence the F-term condition giving rise to the symmetry breaking is invariant under a larger group than the original group $G$, namely the complexification of the group $G^{\mathbb{C}}$. Now let us assume that the symmetry breaking again breaks $G$ to $H$. As we already mentioned, the target space of the nonlinear sigma model must be Kähler [19]. If the coset space $G / H$ is by itself Kähler, then everything is as in the non-supersymmetric case and all the NG bosons of the symmetry breaking are genuine [43, 44]. However it often happens that the coset space $G / H$ is not Kähler. In this case, supersymmetry enforces additional bosons to be massless; they are called quasi-NG bosons [40-42]. It comes back to the complexification of the symmetry. A quasi-NG always comes in a pair with a genuine NG; this is because the chiral superfield is complex and the case of the quasi-NG arises when the broken generator is Hermitian. If for instance we consider an $\mathrm{SU}(2)$ subgroup, where the generators are the three Pauli matrices, an example of quasi-NG bosons is where the generator $\tau^{3}$ is broken, giving 1 genuine NG boson and 1 quasi-NG boson. If on the other hand $\tau^{1}+i \tau^{2}$ is broken (which is non-Hermitian), then we have 2 real genuine NGs. In the first case, the multiplets corresponding to broken Hermitian generators are thus called mixed or M-type, whereas those corresponding to non-Hermitian generators are called pure or P-type. Some conditions for the existence of quasi-NG bosons are known. (1) In the absence of gauge symmetry there must appear at least one M-type, and therefore one quasi-NG boson [45, 46]. (2) When the symmetry $G$ is broken by a VEV belonging to a real representation of $G$, there appear only M-type NGs leading to the same number of quasi-NG bosons as the number of NG bosons, and the target space is $G^{\mathbb{C}} / H^{\mathbb{C}} \simeq T^{*}(G / H)$ [45]. This is actually the case that we need for our purpose. Namely, when chiral symmetry $\mathrm{SU}(N)_{\mathrm{L}} \times \mathrm{SU}(N)_{\mathrm{R}}$ is spontaneously broken down to $\mathrm{SU}(N)_{\mathrm{L}+\mathrm{R}}$, the target space is

$$
\frac{\mathrm{SL}(N, \mathbb{C})_{\mathrm{L}} \times \mathrm{SL}(N, \mathbb{C})_{\mathrm{R}}}{\mathrm{SL}(N, \mathbb{C})_{\mathrm{L}+\mathrm{R}}} \simeq \mathrm{SL}(N, \mathbb{C})
$$


which is the complexification of $\mathrm{SU}(N)$. The most general effective Lagrangian in this case was constructed in ref. [47] and possible higher-derivative terms were constructed on the canonical branch [35].

In this paper, we construct a manifestly supersymmetric Lagrangian in the off-shell superfield formalism, based on an $\operatorname{SL}(N, \mathbb{C})$-valued field. We find that on the canonical branch it gives a Lagrangian with 2 and 4 derivatives. The fourth-order derivative term is however not the Skyrme term; not even when the field is restricted to an $\mathrm{SU}(N)$ submanifold. On the non-canonical branch the situation is different, for which we will work with $\mathrm{SU}(2)$. We are able to write down a manifestly supersymmetric Skyrme-like Lagrangian density in terms of the $\mathrm{SL}(2, \mathbb{C})$-valued field, which however does not take the simple form of the Skyrme term. Nevertheless, if we restrict to a submanifold where the field only takes values in $\mathrm{SU}(2)$, then the fourth-order derivative term reduces exactly to the standard Skyrme term. The quasi-NG bosons therefore change the Lagrangian density (and the target space manifold) compared with the non-supersymmetric case. But once they are turned off, the Skyrme term remains. One peculiarity of the solution on the non-canonical branch, however, is that when turning on a kinetic term (the Dirichlet term), the algebraic solution to the auxiliary field eliminates the kinetic term and leaves only a potential behind. This is, however, a manifestly supersymmetric way to introduce a potential in the "extreme" Skyrme model without adding a superpotential. This was also the case for supersymmetric baby Skyrmions [30, 31, 34, 36]. Our case is actually the first example of a solution on the non-canonical branch in a model with multi-component chiral superfields.

Returning to the problem of four time derivatives. For both the solution on the canonical branch and non-canonical branch, the Lagrangian possesses four time derivatives. However, in the case of the non-canonical branch, when the field is restricted to the $\mathrm{SU}(2)$ submanifold, then the terms with four time derivatives cancel out.

The paper is organized as follows. We set the notation and review the construction of manifestly supersymmetric higher-derivative terms in section 2, both for generic fields and for fields taking values in a coset. Then in section 3 we present our calculation of a supersymmetric Skyrme term as well as the addition of the kinetic term. In section 4 we illustrate the possibility of gauging a global symmetry of the model. Finally we conclude with a discussion and outlook in section 5 .

\section{The formalism}

In this section we briefly review the construction of a supersymmetric higher-derivative term. We consider a higher-derivative Lagrangian that consists of $\mathcal{N}=1$ chiral superfields. We basically follow the Wess-Bagger conventions [48].

\subsection{General action}

The component expansion of the chiral superfield in the $x$-basis is given by

$$
\Phi^{i}(x, \theta, \bar{\theta})=\varphi^{i}+i \theta \sigma^{\mu} \bar{\theta} \partial_{\mu} \varphi^{i}+\frac{1}{4} \theta^{2} \bar{\theta}^{2} \square \varphi^{i}+\theta^{2} F^{i}+\cdots
$$


where $i=1, \ldots, N$ labels the multiple chiral superfields and the ellipses denote terms involving fermions. Here $\varphi_{i}$ are complex scalar fields and $F_{i}$ are auxiliary fields. The sigma matrices are defined as $\sigma^{\mu} \equiv\left(\mathbf{1}_{2}, \vec{\tau}\right)$, where $\vec{\tau}=\left(\tau^{1}, \tau^{2}, \tau^{3}\right)$ are the Pauli matrices. The supercovariant derivatives read

$$
D_{\alpha}=\frac{\partial}{\partial \theta^{\alpha}}+i\left(\sigma^{\mu}\right)_{\alpha \dot{\alpha}} \bar{\theta}^{\dot{\alpha}} \partial_{\mu}, \quad \bar{D}_{\dot{\alpha}}=-\frac{\partial}{\partial \bar{\theta}^{\dot{\alpha}}}-i \theta^{\alpha}\left(\sigma^{\mu}\right)_{\alpha \dot{\alpha}} \partial_{\mu} .
$$

A special combination of chiral superfields yields a higher-derivative Lagrangian density without the auxiliary field problem, which was mentioned in the introduction and can be written as

$$
\frac{1}{16} \int d^{4} \theta \Lambda_{i k \bar{j} \bar{l}}\left(\Phi, \Phi^{\dagger}\right) D^{\alpha} \Phi^{i} D_{\alpha} \Phi^{k} \bar{D}_{\dot{\alpha}} \Phi^{\dagger \bar{j}} \bar{D}^{\dot{\alpha}} \Phi^{\dagger \bar{l}}
$$

The tensor $\Lambda_{i k \bar{j} \bar{l}}$ can be regarded as a $(2,2)$ Kähler tensor, which is symmetric in the two holomorphic indices, $i$ and $k$ and it is also symmetric in the two anti-holomorphic indices $\bar{j}$ and $\bar{l}[35]$. This can be seen from the fact that $D_{\alpha} \Phi^{i}$ transforms like a vector under field redefinitions.

The bosonic components of the term (2.3) are given by

$$
\Lambda_{i k \bar{j} \bar{l}}(\varphi, \bar{\varphi})\left[\left(\partial_{\mu} \varphi^{i} \partial^{\mu} \varphi^{k}\right)\left(\partial_{\nu} \bar{\varphi}^{\bar{j}} \partial^{\nu} \bar{\varphi}^{\bar{l}}\right)-\partial_{\mu} \varphi^{i} F^{k} \partial^{\mu} \bar{\varphi}^{\bar{j}} \bar{F}^{\bar{l}}+F^{i} \bar{F}^{\bar{j}} F^{k} \bar{F}^{\bar{l}}\right] .
$$

The auxiliary field with spacetime derivatives does not appear in these terms. ${ }^{2}$ Then the supersymmetric chiral model with the higher-derivative term is given by

$$
\begin{aligned}
\mathcal{L}= & \int d^{4} \theta K\left(\Phi^{i}, \Phi^{\dagger \bar{j}}\right)+\frac{1}{16} \int d^{4} \theta \Lambda_{i k \bar{j} \bar{l}}\left(\Phi, \Phi^{\dagger}\right) D^{\alpha} \Phi^{i} D_{\alpha} \Phi^{k} \bar{D}_{\dot{\alpha}} \Phi^{\dagger \bar{j}} \bar{D}^{\dot{\alpha}} \Phi^{\dagger \bar{l}} \\
& +\left(\int d^{2} \theta W\left(\Phi^{i}\right)+\text { h.c. }\right)
\end{aligned}
$$

where $K$ is the Kähler potential and $W$ is a superpotential. The equation of motion for the auxiliary field is

$$
\frac{\partial^{2} K}{\partial \varphi^{i} \partial \bar{\varphi}^{\bar{j}}} F^{i}-2 \partial_{\mu} \varphi^{i} F^{k} \Lambda_{i k \bar{j} \bar{l}} \partial^{\mu} \bar{\varphi}^{\bar{l}}+2 \Lambda_{i k \bar{j} \bar{l}} F^{i} F^{k} \bar{F}^{\bar{l}}+\frac{\partial \bar{W}}{\partial \bar{\varphi}_{\bar{j}}}=0 .
$$

Using this equation, we can eliminate the auxiliary fields and calculate the on-shell Lagrangian. Since eq. (2.6) is an algebraic equation of cubic order, it has several solutions and there are distinct branches of on-shell Lagrangians associated with these solutions [49]. Throughout this paper, we consider the $W=0$ case for simplicity. In this case, $F_{i}=0$ is always a solution. For this solution, the on-shell Lagrangian reads

$$
\mathcal{L}_{b}=-\frac{\partial^{2} K}{\partial \varphi^{i} \partial \bar{\varphi}^{\bar{j}}} \partial_{\mu} \varphi^{i} \partial^{\mu} \bar{\varphi}^{\bar{j}}+\Lambda_{i k \bar{j} \bar{l}}\left(\partial_{\mu} \varphi^{i} \partial^{\mu} \varphi^{k}\right)\left(\partial_{\nu} \bar{\varphi}^{\bar{j}} \partial^{\nu} \bar{\varphi}^{\bar{l}}\right) .
$$

We call this the canonical branch. The Lagrangian contains the ordinary kinetic term and a fourth-order derivative term. One can take the small $\Lambda$ limit on the canonical

\footnotetext{
${ }^{2}$ There are auxiliary fields with spacetime derivatives in the fermionic sector. A solution to the auxiliary field equation that contains fermions is obtained order by order in the fermionic fields. In this paper we concentrate on the bosonic sector for which analytic solutions to the auxiliary field equation are found.
} 
branch. Therefore the higher-derivative interactions can be perturbatively introduced to the ordinary (second-order derivative) theory. We note that since the Kähler tensor $\Lambda_{i k \bar{j} \bar{l}}$ is an arbitrary function of the complex scalars $\varphi^{i}$, it can indeed contain terms with arbitrary orders of spacetime derivatives. An example is the scalar part of the $\mathcal{N}=1$ supersymmetric completion of the Dirac-Born-Infeld action for a single D3-brane [50]. Other examples are the supersymmetric Faddeev-Skyrme model [34], the effective action of supersymmetric field theories $[27,28,51-53]$.

In general, there are more solutions than $F_{i}=0$; we call the branch of solutions with $F_{i} \neq 0$ the non-canonical branch. Even though the equation of motion for the auxiliary field is algebraic, it is not so straightforward to find an analytic solution for the noncanonical branch, since the equation is a simultaneous equation of cubic power. For single chiral-superfield models, the analytic solutions to the equation and the on-shell Lagrangian on the non-canonical branch has been found in ref. [34]. For the single chiral-superfield case, the ordinary kinetic term cancels out on the non-canonical branch and the Kähler tensor $\Lambda$ enters into the Lagrangian as $\Lambda^{-1}$. Hence, one cannot take the small $\Lambda$ limit in the on-shell Lagrangian. This means that the higher-derivative interactions are introduced non-perturbatively on the non-canonical branch.

\subsection{Chiral symmetry breaking}

Now we will take the above considerations and apply them to the case of a chiral symmetry breaking of the form

$$
G=\mathrm{SU}(N)_{\mathrm{L}} \times \mathrm{SU}(N)_{\mathrm{R}} \rightarrow H=\mathrm{SU}(N)_{\mathrm{L}+\mathrm{R}},
$$

giving the corresponding coset

$$
G / H=\frac{\mathrm{SU}(N)_{\mathrm{L}} \times \mathrm{SU}(N)_{\mathrm{R}}}{\mathrm{SU}(N)_{\mathrm{L}+\mathrm{R}}} \simeq \mathrm{SU}(N),
$$

which is spanned by NG modes. As we mentioned in the introduction, in the supersymmetric case, the group $G$ is complexified and the group $H$ is changed to the so-called complex isotropy group, see e.g. ref. [35]. This means that the target space relevant for the supersymmetric nonlinear sigma model describing the symmetry breaking is

$$
G^{\mathbb{C}} / \hat{H} \simeq \mathrm{SU}(N)^{\mathbb{C}}=G^{\mathbb{C}} / H^{\mathbb{C}} \simeq \mathrm{SL}(N, \mathbb{C}) \simeq T^{*} \mathrm{SU}(N) .
$$

The fact that $\hat{H}=H^{\mathbb{C}}$ here (which is not the case in general), is because the realization of quasi-NG bosons is a so-called maximal realization or fully-doubled realization [40-42, 45].

We now represent the coset with the following nonlinear sigma model field

$$
M=\exp \left(i \Phi^{A} t^{A}\right) \in G^{\mathbb{C}} / \hat{H},
$$

where the NG superfields take the form

$$
\Phi^{A}(y, \theta)=\pi^{A}(y)+i \sigma^{A}(y)+\theta \psi^{A}(y)+\theta^{2} F^{A}(y),
$$


where $t^{A}$ are the generators of $G^{\mathbb{C}} / \hat{H}, \pi^{A}$ are (genuine) NG bosons, $\sigma^{A}$ are quasi-NG bosons, $\psi^{A}$ are quasi-NG fermions and finally, $F^{A}$ are auxiliary fields.

The NG supermultiplet obeys the following nonlinear transformation law

$$
M \rightarrow M^{\prime}=g_{\mathrm{L}} M g_{\mathrm{R}}, \quad\left(g_{\mathrm{L}}, g_{\mathrm{R}}\right) \in \mathrm{SU}(N)_{\mathrm{L}} \times \mathrm{SU}(N)_{\mathrm{R}}
$$

This yields the transformations

$$
M M^{\dagger} \rightarrow g_{\mathrm{L}} M M^{\dagger} g_{\mathrm{L}}^{\dagger}
$$

implying that the Kähler potential can be constructed as a function of $\operatorname{Tr}\left(M M^{\dagger}\right)$. The simplest example is

$$
K_{0}=f_{\pi}^{2} \operatorname{Tr}\left(M M^{\dagger}\right)
$$

leading to the free bosonic Lagrangian density

$$
\mathcal{L}_{0, b}^{(2)}=-f_{\pi}^{2} \operatorname{Tr}\left(\partial_{\mu} M \partial^{\mu} M^{\dagger}\right),
$$

where $M$ in the last equation is the lowest component of the NG multiplet with the same symbol. The Kähler potential (2.15) is, however, not general; the most general Kähler potential can be written as [47]

$$
K=f\left[\operatorname{Tr}\left[M M^{\dagger}\right], \operatorname{Tr}\left[\left(M M^{\dagger}\right)^{2}\right], \cdots, \operatorname{Tr}\left[\left(M M^{\dagger}\right)^{N-1}\right]\right],
$$

with an arbitrary functional of the $N-1$ variables. Physically, the reason why the function can be arbitrary is due to the existence of the quasi-NG bosons [54-57]. The isometry of the target space is $G$ and not its complexification $G^{\mathbb{C}}$ and hence the target manifold is not homogeneous. The shape of the manifold can be deformed along the directions of the quasi-NG bosons, keeping the isometry $G$. By setting the quasi-NG bosons to zero, we restrict to a submanifold

$$
U=\left.M\right|_{\sigma^{A}=0} \in \mathrm{SU}(N)
$$

which in turn simplifies the kinetic term from the Kähler potential to the usual chiral Lagrangian

$$
\left.\mathcal{L}_{0, b}^{(2)}\right|_{\sigma^{A}=0}=-f_{\pi}^{2} \operatorname{Tr}\left(\partial_{\mu} U \partial^{\mu} U^{\dagger}\right),
$$

where $f_{\pi}$ and $f$ are related.

\section{The supersymmetric Skyrme term}

In this section we consider a fourth-order derivative term based on the formalism presented in the last section. 


\subsection{A fourth-order derivative term in the chiral Lagrangian}

The fourth-order derivative term that we consider is of the form

$$
\begin{aligned}
\mathcal{L}_{0}^{(4)} & =\frac{1}{16} \int d^{4} \theta \Lambda_{i k \bar{j} \bar{l}}\left(\Phi, \Phi^{\dagger}\right) D^{\alpha} \Phi^{i} D_{\alpha} \Phi^{k} \bar{D}_{\dot{\alpha}} \Phi^{\bar{j} \dagger} \bar{D}^{\dot{\alpha}} \Phi^{\bar{l} \dagger} \\
& =\frac{1}{16} \int d^{4} \theta \Lambda\left(M, M^{\dagger}\right) \operatorname{Tr}\left[D^{\alpha} M \bar{D}_{\dot{\alpha}} M^{\dagger} D_{\alpha} M \bar{D}^{\dot{\alpha}} M^{\dagger}\right],
\end{aligned}
$$

which is the simplest candidate for a fourth-order derivative term [35] and $\Lambda_{i k \bar{j} \bar{l}}$ is a $G$ covariant $(2,2)$ Kähler tensor determined from the right-hand side of the above equation. $\Lambda\left(M, M^{\dagger}\right)$ is a $G$-invariant real scalar on the target space, given by

$$
\Lambda\left(M, M^{\dagger}\right)=g\left[\operatorname{Tr}\left[M M^{\dagger}\right], \operatorname{Tr}\left[\left(M M^{\dagger}\right)^{2}\right], \cdots, \operatorname{Tr}\left[\left(M M^{\dagger}\right)^{N-1}\right]\right]
$$

with an arbitrary function $g$ of $N-1$ variables. It is also possible to consider a non- $G$ invariant function for $\Lambda\left(M, M^{\dagger}\right)$ such as $\Lambda\left(M, M^{\dagger}\right)=\operatorname{Tr}\left(M+M^{\dagger}\right)$.

The term (3.1) is of course not the most general term with four derivatives, avoiding the auxiliary field problem, that can be written down, see e.g. ref. [35] for more general four-derivative terms. The bosonic part of the term reads

$$
\mathcal{L}_{0, b}^{(4)}=\Lambda\left(M, M^{\dagger}\right) \operatorname{Tr}\left[M_{\mu}^{\dagger} M_{\nu} M^{\mu \dagger} M^{\nu}+\left(F^{\dagger} F\right)^{2}-M_{\mu}^{\dagger} M^{\mu} F^{\dagger} F-M_{\mu} M^{\mu \dagger} F F^{\dagger}\right]
$$

where we have introduced the notation $M_{\mu} \equiv \partial_{\mu} M$, which we will use throughout the paper.

\subsection{Canonical branch}

We can now construct a theory with second-order and fourth-order derivative terms, for instance, by adding the two terms (2.16) and (3.3)

$$
\mathcal{L}_{0}=\mathcal{L}_{0}^{(2)}+\mathcal{L}_{0}^{(4)}
$$

As we do not consider adding superpotentials to the theory in this paper, the simplest solution to $F$ is given by putting $F$ on the canonical branch: $F=0$, giving

$$
\mathcal{L}_{0, b}=-f_{\pi}^{2} \operatorname{Tr} M_{\mu} M^{\mu \dagger}+\Lambda\left(M, M^{\dagger}\right) \operatorname{Tr} M_{\mu}^{\dagger} M_{\nu} M^{\mu \dagger} M^{\nu} .
$$

Comparing the bosonic part of this theory with the Skyrme model, the fourth-order derivative term is clearly different from the Skyrme term. First of all, it is not a curvature term (i.e. cannot be written as a curvature of a tensor) and second of all, it contains four time derivatives and hence suffers from the Ostrogradsky instability [58]. As we mentioned already in the introduction, this feature is typical for supersymmetric higher-derivative theories. 


\subsection{Non-canonical branch: a supersymmetric Skyrme term}

As is clear from the discussion, there is also the possibility of considering the non-canonical branch of solutions for the auxiliary fields, i.e. $F \neq 0$. For non-Abelian valued superfields, no explicit solutions to the auxiliary field equation has been found so far, even for single field models. A prototypical example of such a non-Abelian valued field is the Skyrme field. We will explicitly construct the first solution for models with non-Abelian valued fields. Let us start with the case in the absence of the kinetic term.

The equation of motion for the auxiliary field coming from the Lagrangian density (3.3) reads

$$
2 F F^{\dagger} F-F M_{\mu}^{\dagger} M^{\mu}-M_{\mu} M^{\mu \dagger} F=0,
$$

when varied with respect to $F^{\dagger}$ and

$$
2 F^{\dagger} F F^{\dagger}-M_{\mu}^{\dagger} M^{\mu} F^{\dagger}-F^{\dagger} M_{\mu} M^{\mu \dagger}=0 .
$$

when varied with respect to $F$, which is simply the complex conjugate of eq. (3.6). We have assumed that $\Lambda\left(M, M^{\dagger}\right) \neq 0$, which multiplies the whole equation (the $\Lambda\left(M, M^{\dagger}\right)=0$ solution is just turning off the fourth-order derivative term).

Multiplying (3.6) by $F^{-1}$ from the right and (3.7) by $\left(F^{\dagger}\right)^{-1}$ from the left gives us

$$
\begin{aligned}
2 F F^{\dagger}-F M_{\mu}^{\dagger} M^{\mu} F^{-1}-M_{\mu} M^{\mu \dagger} & =0, \\
2 F F^{\dagger}-\left(F^{\dagger}\right)^{-1} M_{\mu}^{\dagger} M^{\mu} F^{\dagger}-M_{\mu} M^{\mu \dagger} & =0 .
\end{aligned}
$$

Subtracting (3.9) from (3.8), we get

$$
\left[M_{\mu}^{\dagger} M^{\mu}, F^{\dagger} F\right]=0
$$

This means that they are diagonalizable in the same basis.

Now we do almost the same, but for $F^{\dagger} F$. Multiplying (3.6) by $F^{-1}$ from the left and (3.7) by $\left(F^{\dagger}\right)^{-1}$ from the right gives us now

$$
\begin{aligned}
2 F^{\dagger} F-M_{\mu}^{\dagger} M^{\mu}-F^{-1} M_{\mu} M^{\mu \dagger} F & =0, \\
2 F^{\dagger} F-M_{\mu}^{\dagger} M^{\mu}-F^{\dagger} M_{\mu} M^{\mu \dagger}\left(F^{\dagger}\right)^{-1} & =0 .
\end{aligned}
$$

Subtracting now (3.12) from (3.11), we get

$$
\left[M_{\mu} M^{\mu \dagger}, F F^{\dagger}\right]=0 .
$$

This means that these two matrices are also diagonalizable in the same basis.

A Hermitian matrix $W$ can be written as

$$
W=U D U^{\dagger},
$$

where $U$ is a special unitary matrix and $D$ is a diagonal matrix composed of the real eigenvalues of $W$ in its diagonal. 
We will now write the auxiliary field as

$$
F=A R B,
$$

where as we will see shortly, $R$ is a diagonal matrix except for when some of the eigenvalues are degenerate, and $A$ is a special unitary matrix that diagonalizes $F F^{\dagger}$ :

$$
F F^{\dagger}=A R R^{\dagger} A^{\dagger},
$$

while $B$ is a special unitary matrix that diagonalizes $F^{\dagger} F$ :

$$
F^{\dagger} F=B^{\dagger} R^{\dagger} R B
$$

This means that $R R^{\dagger}$ and $R^{\dagger} R$ are both diagonal matrices.

The eigenvalues of both $F F^{\dagger}$ and $F^{\dagger} F$ must be the same due to Sylvester's determinant theorem:

$$
\operatorname{det}\left(\lambda \mathbf{1}-F^{\dagger} F\right)=\operatorname{det}\left(\lambda \mathbf{1}-F F^{\dagger}\right),
$$

but this does not prove that $R^{\dagger} R=R R^{\dagger}$ (that is, it does not prove that the eigenvalues appear in the same order along the diagonal). ${ }^{3}$

Let us simplify the problem for the moment and consider $N=2$, namely $\operatorname{SL}(2, \mathbb{C})$. We know from (3.18) that the two eigenvalues of $F^{\dagger} F$ are equal to the two of $F F^{\dagger}$. Both these matrices are Hermitian and thus both eigenvalues are real (and positive semi-definite, due to the fact that they are composed of a matrix multiplied by its Hermitian conjugate). Let us set

$$
R=\left(\begin{array}{ll}
\alpha & \beta \\
\gamma & \delta
\end{array}\right), \quad \alpha, \beta, \gamma, \delta \in \mathbb{C},
$$

and require that $R R^{\dagger}$ is diagonal. That translates to the following condition

$$
\bar{\beta}=-\frac{\gamma \bar{\alpha}}{\delta},
$$

while the condition that $R^{\dagger} R$ is diagonal translates to

$$
\bar{\beta}=-\frac{\gamma \bar{\delta}}{\alpha},
$$

both constraints are happily satisfied with $\gamma=\beta=0$ and thus $R$ is itself a diagonal matrix. In this case, it is obvious that $R R^{\dagger}=R^{\dagger} R$ and the eigenvalues must be positive.

Let us now assume that $\beta \neq 0$ which means in turn that $\gamma \neq 0$. The two constraints (3.20) and (3.21) can be solved by

$$
R=\left(\begin{array}{cc}
c e^{i \eta}-\bar{\gamma} e^{i(\eta+\theta)} \\
\gamma & c e^{i \theta}
\end{array}\right), \quad \gamma \in \mathbb{C}^{*}, \quad c, \eta, \theta \in \mathbb{R} .
$$

\footnotetext{
${ }^{3}$ If both $A$ and $B$ are prepared as column vectors of eigenvectors, then switching the order of some of the eigenvectors in $A$ but not in $B$ renders $R^{\dagger} R \neq R R^{\dagger}$, even though they are both diagonal matrices with the same real eigenvalues.
} 
Now since $\operatorname{det} F=1=\operatorname{det} A \operatorname{det} R \operatorname{det} B=\operatorname{det} R$, we have

$$
\operatorname{det} R=c^{2} e^{i(\eta+\theta)}+|\gamma|^{2} e^{i(\eta+\theta)}=1,
$$

which is solved by $c=\sqrt{1-|\gamma|^{2}}$ and $\theta=-\eta$. We now have

$$
R=\left(\begin{array}{cc}
\sqrt{1-|\gamma|^{2}} e^{i \eta} & -\bar{\gamma} \\
\gamma & \sqrt{1-|\gamma|^{2}} e^{-i \eta}
\end{array}\right), \quad \gamma \in \mathbb{C}^{*}, 0<|\gamma|<1, \quad \eta \in \mathbb{R} .
$$

Now we have

$$
R^{\dagger} R=R R^{\dagger}=\mathbf{1}_{2},
$$

which means that again $R^{\dagger} R=R R^{\dagger}$ (but now they both additionally have degenerate eigenvalues). We do not have a proof for $N>2$, but let us consider $N=2$ for now.

Let us now consider (3.8) and (3.9) with (3.15) which read

$$
\begin{aligned}
2 R R^{\dagger}-R B M_{\mu}^{\dagger} M^{\mu} B^{\dagger} R^{-1}-A^{\dagger} M_{\mu} M^{\mu \dagger} A & =0, \\
2 R R^{\dagger}-\left(R^{\dagger}\right)^{-1} B M_{\mu}^{\dagger} M^{\mu} B^{\dagger} R^{\dagger}-A^{\dagger} M_{\mu} M^{\mu \dagger} A & =0 .
\end{aligned}
$$

If $R$ is diagonal, then the above equations simplify as

$$
R B M_{\mu}^{\dagger} M^{\mu} B^{\dagger} R^{-1}=B M_{\mu}^{\dagger} M^{\mu} B^{\dagger},
$$

and

$$
\left(R^{\dagger}\right)^{-1} B M_{\mu}^{\dagger} M^{\mu} B^{\dagger} R^{\dagger}=B M_{\mu}^{\dagger} M^{\mu} B^{\dagger} .
$$

However, we should first consider the case that $R$ is not a diagonal matrix, which is the case where it takes the form (3.24). Let us consider eq. (3.26), which now reads

$$
\begin{aligned}
2 \mathbf{1}_{2} & -\left(\begin{array}{cc}
\sqrt{1-|\gamma|^{2}} e^{i \eta} & -\bar{\gamma} \\
\gamma & \sqrt{1-|\gamma|^{2}} e^{-i \eta}
\end{array}\right)\left(\begin{array}{cc}
m_{1} \\
m_{2}
\end{array}\right)\left(\begin{array}{cc}
\sqrt{1-|\gamma|^{2}} e^{-i \eta} & \bar{\gamma} \\
-\gamma & \sqrt{1-|\gamma|^{2}} e^{i \eta}
\end{array}\right) \\
& -\left(\begin{array}{cc}
\tilde{m}_{1} & \\
\tilde{m}_{2}
\end{array}\right)=\left(\begin{array}{cc}
* & -\bar{\gamma} \sqrt{1-|\gamma|^{2}}\left(m_{1}-m_{2}\right) e^{i \eta} \\
-\gamma \sqrt{1-|\gamma|^{2}}\left(m_{1}-m_{2}\right) e^{-i \eta} & *
\end{array}\right)=0 .
\end{aligned}
$$

There are now three possibilities. If $m_{1}=m_{2}$, then $B M_{\mu}^{\dagger} M^{\mu} B^{\dagger}$ is proportional to the unit matrix $\mathbf{1}_{2}$ and so the simplification (3.28) holds. The two remaining options are $\gamma=0$ and $\gamma=e^{i \tau}$ with $\tau \in \mathbb{R}$ a real phase. In the first case, $R$ is diagonal and so the simplification (3.28) holds again. If, however, $|\gamma|=1$ then the situation corresponds to switching the two eigenvalues of $M_{\mu}^{\dagger} M^{\mu}$ but not of $M_{\mu} M^{\mu \dagger}$; this can be done easily by switching the order of the eigenvectors in $B$, but not in $A$. Thus again we can use the simplification (3.28). Using instead eq. (3.11), we can show the analogous case for $\tilde{m}_{1}$ and $\tilde{m}_{2}$. Therefore the simplification (3.29) holds as well.

Since we have proven that the simplifications (3.28) and (3.29) hold, we can now write

$$
\begin{aligned}
& 2 R R^{\dagger}-B M_{\mu}^{\dagger} M^{\mu} B^{\dagger}-A^{\dagger} M_{\mu} M^{\mu \dagger} A=0, \\
& 2 R^{\dagger} R-B M_{\mu}^{\dagger} M^{\mu} B^{\dagger}-A^{\dagger} M_{\mu} M^{\mu \dagger} A=0,
\end{aligned}
$$


or equivalently

$$
\begin{aligned}
& 2 F F^{\dagger}-A B M_{\mu}^{\dagger} M^{\mu} B^{\dagger} A^{\dagger}-M_{\mu} M^{\mu \dagger}=0 . \\
& 2 F^{\dagger} F-M_{\mu}^{\dagger} M^{\mu}-B^{\dagger} A^{\dagger} M_{\mu} M^{\mu \dagger} A B=0 .
\end{aligned}
$$

Eqs. (3.31) and (3.32) clearly show that $R R^{\dagger}=R^{\dagger} R$. Eq. (3.33) shows that $F F^{\dagger}$ is equal to the average of $M_{\mu} M^{\mu \dagger}$ and $M_{\mu}^{\dagger} M^{\mu}$ rotated twice (by two consecutive special unitary transformations). Similarly for $F^{\dagger} F$.

Inserting (3.33) and (3.34) into (3.3), we get

$$
\begin{aligned}
\mathcal{L}_{0, b}^{(4)}= & \Lambda\left(M, M^{\dagger}\right) \operatorname{Tr}\left[M_{\mu}^{\dagger} M_{\nu} M^{\mu \dagger} M^{\nu}-\frac{1}{4} M_{\mu}^{\dagger} M^{\mu} M_{\nu}^{\dagger} M^{\nu}-\frac{1}{4} M_{\mu} M^{\mu \dagger} M_{\nu} M^{\nu \dagger}\right. \\
& \left.-\frac{1}{2} A^{\dagger} M_{\mu} M^{\mu \dagger} A B M_{\nu}^{\dagger} M^{\nu} B^{\dagger}\right] .
\end{aligned}
$$

Note that both $A^{\dagger} M_{\mu} M^{\mu \dagger} A$ and $B M_{\nu}^{\dagger} M^{\nu} B^{\dagger}$ are diagonal matrices and that

$$
\operatorname{Tr}\left[A^{\dagger} M_{\mu} M^{\mu \dagger} A B M_{\nu}^{\dagger} M^{\nu} B^{\dagger}\right]=\operatorname{Tr}\left(\begin{array}{cc}
m_{1} & \\
& m_{2}
\end{array}\right)\left(\begin{array}{cc}
\tilde{m}_{1} & \\
& \tilde{m}_{2}
\end{array}\right)=m_{1} \tilde{m}_{1}+m_{2} \tilde{m}_{2} .
$$

For $\operatorname{SL}(2, \mathbb{C})$, it is possible to write the eigenvalue equation as

$$
\begin{aligned}
0 & =\lambda^{2}-\lambda \operatorname{Tr}\left[A^{\dagger} M_{\mu} M^{\mu \dagger} A\right]+\operatorname{det}\left[A^{\dagger} M_{\mu} M^{\mu \dagger} A\right] \\
& =\lambda^{2}-\lambda \operatorname{Tr}\left[M_{\mu} M^{\mu \dagger}\right]+\operatorname{det}\left[M_{\mu} M^{\mu \dagger}\right] \\
& =\lambda^{2}-\lambda \operatorname{Tr}\left[M_{\mu} M^{\mu \dagger}\right]+\frac{1}{2} \operatorname{Tr}\left[M_{\mu} M^{\mu \dagger}\right]^{2}-\frac{1}{2} \operatorname{Tr}\left[M_{\mu} M^{\mu \dagger} M_{\nu} M^{\nu \dagger}\right]
\end{aligned}
$$

where we have used the Cayley-Hamilton theorem, $\operatorname{det} A=\frac{1}{2}\left[(\operatorname{Tr} A)^{2}-\operatorname{Tr} A^{2}\right]$, in the last line. We can therefore write

$$
\begin{aligned}
\operatorname{Tr} & {\left[A^{\dagger} M_{\mu} M^{\mu \dagger} A B M_{\nu}^{\dagger} M^{\nu} B^{\dagger}\right]=\frac{1}{2}\left(\operatorname{Tr}\left[M_{\mu} M^{\mu \dagger}\right]\right)^{2} } \\
& \pm \sqrt{\left(\operatorname{Tr}\left[M_{\mu}^{\dagger} M^{\mu} M_{\nu}^{\dagger} M^{\nu}\right]-\frac{1}{2}\left(\operatorname{Tr}\left[M_{\mu} M^{\mu \dagger}\right]\right)^{2}\right)\left(\operatorname{Tr}\left[M_{\mu} M^{\mu \dagger} M_{\nu} M^{\nu \dagger}\right]-\frac{1}{2}\left(\operatorname{Tr}\left[M_{\mu} M^{\mu \dagger}\right]\right)^{2}\right)} .
\end{aligned}
$$

The sign ambiguity depends on the order of the eigenvalues (i.e. holding $m_{1}$ and $m_{2}$ fixed and switching $\tilde{m}_{1}$ and $\tilde{m}_{2}$; this can be done as already mentioned by switching the eigenvectors in $A$ but not in $B$ ).

Putting the pieces together, we can write down the Lagrangian density for the full fourth-order derivative term as

$$
\begin{aligned}
\mathcal{L}_{0, b}^{(4)}= & \frac{\Lambda\left(M, M^{\dagger}\right)}{2}\left\{\operatorname{Tr}\left[2 M_{\mu}^{\dagger} M_{\nu} M^{\mu \dagger} M^{\nu}-\frac{1}{2} M_{\mu}^{\dagger} M^{\mu} M_{\nu}^{\dagger} M^{\nu}-\frac{1}{2} M_{\mu} M^{\mu \dagger} M_{\nu} M^{\nu \dagger}\right]\right. \\
& -\frac{1}{2}\left(\operatorname{Tr}\left[M_{\mu} M^{\mu \dagger}\right]\right)^{2} \\
& \mp \sqrt{\left(\operatorname{Tr}\left[M_{\mu}^{\dagger} M^{\mu} M_{\nu}^{\dagger} M^{\nu}\right]-\frac{1}{2}\left(\operatorname{Tr}\left[M_{\mu} M^{\mu \dagger}\right]\right)^{2}\right)\left(\operatorname{Tr}\left[M_{\mu} M^{\mu \dagger} M_{\nu} M^{\nu \dagger}\right]-\frac{1}{2}\left(\operatorname{Tr}\left[M_{\mu} M^{\mu \dagger}\right]\right)^{2}\right)} .
\end{aligned}
$$


Let us consider the four time derivatives term, which is present in the Lagrangian density. For convenience, we consider the form of the Lagrangian density in eq. (3.35) and thus we have

$$
\frac{1}{2} \Lambda\left(M, M^{\dagger}\right) \operatorname{Tr}\left[M_{0}^{\dagger} M^{0}\left(M_{0}^{\dagger} M^{0}-B^{\dagger} A^{\dagger} M_{0} M^{0 \dagger} A B\right)\right],
$$

which means that the term does not cancel out due to the mismatch between $M_{\mu}^{\dagger} M^{\mu}$ and $M_{\mu} M^{\mu \dagger}$ being diagonalizable in the same basis $\left(A\right.$ is generically not equal to $B^{\dagger}$ ). One would expect this term to be a small four time derivative term compared with that coming from the canonical branch, see eq. (3.5). Nevertheless, the above term is present in the Lagrangian and thus gives rise to the Ostrogradsky instability [58].

Now we want to show that we can reduce the Lagrangian density by restricting the field $M \in \mathrm{SL}(2, \mathbb{C})$ to $U \in \mathrm{SU}(2)$, i.e. a unitary field; that is, $M=U$ for which we now have $U^{\dagger} U=U U^{\dagger}=\mathbf{1}_{2}$ and so

$$
U_{\mu}^{\dagger} U=-U^{\dagger} U_{\mu}
$$

This physically means that we turned off the quasi-NG bosons and consider the submanifold spanned only by the genuine NG bosons. We first want to show that the eigenvalues of $U_{\mu}^{\dagger} U^{\mu}$ are the same as those of $U_{\mu} U^{\mu \dagger}$. Hence, we have

$$
\begin{aligned}
& \operatorname{det}\left[\lambda \mathbf{1}_{2}-U_{\mu}^{\dagger} U^{\mu}\right] \\
& =\operatorname{det}\left[\lambda \mathbf{1}_{2}-U_{\mu}^{\dagger} U U^{\dagger} U^{\mu}\right] \\
& =\operatorname{det}\left[\lambda \mathbf{1}_{2}-U^{\dagger} U_{\mu} U^{\mu \dagger} U\right] \\
& =\operatorname{det}\left[U^{\dagger}\left(\lambda \mathbf{1}_{2}-U_{\mu} U^{\mu \dagger}\right) U\right] \\
& =\operatorname{det} U^{\dagger} \operatorname{det}\left[\lambda \mathbf{1}_{2}-U_{\mu} U^{\mu \dagger}\right] \operatorname{det} U \\
& =\operatorname{det}\left[\lambda \mathbf{1}_{2}-U_{\mu} U^{\mu \dagger}\right] .
\end{aligned}
$$

This is a great simplification over the case with $M_{\mu}^{\dagger} M^{\mu}$, which in general does not have the same eigenvalues as $M_{\mu} M^{\mu \dagger}$. Continuing, we can write the diagonal matrix

$$
B U_{\mu}^{\dagger} U^{\mu} B^{\dagger}=B U_{\mu}^{\dagger} U U^{\dagger} U^{\mu} B^{\dagger}=B U^{\dagger} U_{\mu} U^{\mu \dagger} U B^{\dagger},
$$

and thus we found a matrix that diagonalizes $U_{\mu} U^{\mu \dagger}$, namely $A=U B^{\dagger}$. We can now calculate the last term in eq. (3.35) as

$$
\operatorname{Tr}\left[A^{\dagger} U_{\mu} U^{\mu \dagger} A B U_{\nu}^{\dagger} U^{\nu} B^{\dagger}\right]=\operatorname{Tr}\left[U^{\dagger} U_{\mu} U^{\mu \dagger} U U_{\nu}^{\dagger} U^{\nu}\right]=\operatorname{Tr}\left[U_{\mu}^{\dagger} U^{\mu} U_{\nu}^{\dagger} U^{\nu}\right] .
$$

It is straightforward to show that

$$
\begin{aligned}
& \operatorname{Tr}\left[U_{\mu} U^{\mu \dagger} U_{\nu} U^{\nu \dagger}\right] \\
& =\operatorname{Tr}\left[U_{\mu} U^{\dagger} U U^{\mu \dagger} U_{\nu} U^{\dagger} U U^{\nu \dagger}\right] \\
& =\operatorname{Tr}\left[U U_{\mu}^{\dagger} U^{\mu} U^{\dagger} U U_{\nu}^{\dagger} U^{\nu} U^{\dagger}\right] \\
& =\operatorname{Tr}\left[U_{\mu}^{\dagger} U^{\mu} U_{\nu}^{\dagger} U^{\nu}\right] .
\end{aligned}
$$


Using now eqs. (3.44) and (3.45), we can write the bosonic Lagrangian density (3.35) for the restricted submanifold spanned only by NG bosons, as

$$
\mathcal{L}_{0, b}^{(4)}=\Lambda\left(U, U^{\dagger}\right) \operatorname{Tr}\left[U_{\mu}^{\dagger} U_{\nu} U^{\mu \dagger} U^{\nu}-U_{\mu}^{\dagger} U^{\mu} U_{\nu}^{\dagger} U^{\nu}\right]
$$

which for $\Lambda\left(U, U^{\dagger}\right)=$ const. is exactly the Skyrme term.

We will now, for consistency, show that the same result follows from the Lagrangian density (3.39). It follows simply by using eq. (3.45) and choosing the upper sign (which means that the eigenvalues are not swapped), for which the last two terms of the Lagrangian density combine to

$$
\frac{\Lambda\left(U, U^{\dagger}\right)}{2} \operatorname{Tr}\left[U_{\mu}^{\dagger} U^{\mu} U_{\nu}^{\dagger} U^{\nu}\right]
$$

and the final result (3.46) again follows.

\subsection{The Dirichlet term}

Now we add the normal kinetic term - the Dirichlet energy — to the Lagrangian, which reads

$$
\mathcal{L}=f_{\pi}^{2} \int d^{4} \theta \operatorname{Tr}\left[M^{\dagger} M\right]+\frac{1}{16} \int d^{4} \theta \Lambda\left(M, M^{\dagger}\right) \operatorname{Tr}\left[\bar{D}_{\dot{\alpha}} M^{\dagger} D_{\alpha} M \bar{D}^{\dot{\alpha}} M^{\dagger} D^{\alpha} M\right] .
$$

The corresponding bosonic Lagrangian reads

$$
\begin{aligned}
\mathcal{L}_{b}= & f_{\pi}^{2} \operatorname{Tr}\left[-M_{\mu}^{\dagger} M^{\mu}+F^{\dagger} F\right] \\
& +\Lambda\left(M, M^{\dagger}\right) \operatorname{Tr}\left[M_{\mu}^{\dagger} M_{\nu} M^{\dagger \mu} M^{\nu}+\left(F^{\dagger} F\right)^{2}-M_{\mu}^{\dagger} M^{\mu} F^{\dagger} F-M_{\mu} M^{\dagger \mu} F F^{\dagger}\right]
\end{aligned}
$$

giving rise to the equations of motion for the auxiliary fields

$$
\begin{aligned}
f_{\pi}^{2} \Lambda^{-1}\left(M, M^{\dagger}\right) F+2 F F^{\dagger} F-F M_{\mu}^{\dagger} M^{\mu}-M_{\mu} M^{\dagger \mu} F & =0, \\
f_{\pi}^{2} \Lambda^{-1}\left(M, M^{\dagger}\right) F^{\dagger}+2 F^{\dagger} F F^{\dagger}-M_{\mu}^{\dagger} M^{\mu} F^{\dagger}-F^{\dagger} M_{\mu} M^{\dagger \mu} & =0 .
\end{aligned}
$$

For a trivial solution $F=0$, the Lagrangian on the canonical branch is given in eq. (3.5).

Next, we study the non-canonical branch associated with an $F \neq 0$ solution. As in the case of the previous section, the equation (3.50) implies that $\left[M_{\mu}^{\dagger} M, F^{\dagger} F\right]=0$. Therefore $M_{\mu}^{\dagger} M^{\mu}$ and $F^{\dagger} F$ are again simultaneously diagonalizable. The same is true for $M_{\mu} M^{\dagger \mu}$ and $F F^{\dagger}$. We can thus proceed along the same lines as the discussion in the previous section, even for the case where the ordinary kinetic term is turned on. The $F \neq 0$ solution for $G^{\mathbb{C}}=\mathrm{SL}(2, \mathbb{C})$ reads

$$
\begin{aligned}
& F F^{\dagger}=\frac{1}{2}\left[A B M_{\mu}^{\dagger} M^{\mu} B^{\dagger} A^{\dagger}+M_{\mu} M^{\dagger \mu}-f_{\pi}^{2} \Lambda^{-1} \mathbf{1}_{2}\right] \\
& F^{\dagger} F=\frac{1}{2}\left[B^{\dagger} A^{\dagger} M_{\mu} M^{\dagger \mu} A B+M_{\mu}^{\dagger} M^{\mu}-f_{\pi}^{2} \Lambda^{-1} \mathbf{1}_{2}\right] .
\end{aligned}
$$


Eliminating the auxiliary fields by using the above solutions, we find that the bosonic part of the Lagrangian on the non-canonical branch is given by

$$
\begin{aligned}
\mathcal{L}_{b}= & \Lambda\left(M, M^{\dagger}\right) \operatorname{Tr}\left[M_{\mu}^{\dagger} M_{\nu} M^{\dagger \mu} M^{\nu}-\frac{1}{4}\left(M_{\mu}^{\dagger} M^{\mu}\right)^{2}-\frac{1}{4}\left(M_{\mu} M^{\dagger \mu}\right)^{2}\right. \\
& \left.-\frac{1}{2} A^{\dagger} M_{\mu} M^{\dagger \mu} A B M_{\nu}^{\dagger} M^{\nu} B^{\dagger}-\frac{f_{\pi}^{2}}{4 \Lambda\left(M, M^{\dagger}\right)} \mathbf{1}_{2}\right] .
\end{aligned}
$$

We note that the ordinary kinetic term cancels out and only the fourth-order derivative term remains along with a potential-like term which is proportional to $\operatorname{Tr} \mathbf{1}_{2}$; this term is absent when $\operatorname{Tr} M^{\dagger} M$ term is not included. This is an alternative way of introducing a potential term into supersymmetric theories without the superpotential [59,60]. The same structure has been found in the $N=1$ case [34]. The term $\operatorname{Tr}\left[A^{\dagger} M_{\mu} M^{\dagger \mu} A B M_{\nu}^{\dagger} M^{\nu} B^{\dagger}\right]$ is evaluated as in section 3 and the first four terms can again be written explicitly as in eq. (3.39).

We now restrict the field to $M=U \in \mathrm{SU}(2)$, as was done in the previous section. The Lagrangian then reduces to

$$
\left.\mathcal{L}_{b}\right|_{M=U}=\Lambda\left(U, U^{\dagger}\right) \operatorname{Tr}\left[U_{\mu}^{\dagger} U_{\nu} U^{\dagger \mu} U^{\nu}-U_{\mu}^{\dagger} U^{\mu} U_{\nu}^{\dagger} U^{\nu}\right]-\operatorname{Tr}\left[\frac{f_{\pi}^{2}}{4 \Lambda\left(U, U^{\dagger}\right)} \mathbf{1}_{2}\right] .
$$

When we consider a $G$-invariant function $\Lambda$, the second term gives just a constant, this is, simply the Skyrme term remains. If we consider a $G$-variant function $\Lambda$ such as $\Lambda=$ $\operatorname{Tr}\left(M+M^{\dagger}\right)$, the second term gives a potential term (but it also breaks the $G$-invariance of the Skyrme term).

\section{Gauging the global symmetry}

Finally, in this section, we point out that we can gauge a global symmetry of the model and write down the interactions of the gauge field. We consider the $\mathrm{SU}(N)_{\mathrm{L}} \times \mathrm{SU}(N)_{\mathrm{R}}$ as a global symmetry of the Skyrme field $M$. We introduce the $\mathcal{N}=1$ vector multiplets $V_{\mathrm{L}}$, $V_{\mathrm{R}}$ associated with $\mathrm{SU}(N)_{\mathrm{L}}$ and $\mathrm{SU}(N)_{\mathrm{R}}$ gauge groups respectively. The vector multiplets are introduced in the gauge covariantized supercovariant derivative which is defined by

$$
\mathcal{D}_{\alpha} M=D_{\alpha} M+\Gamma_{\alpha}^{\mathrm{L}} M+M \Gamma_{\alpha}^{\mathrm{R}}
$$

where $\Gamma_{\alpha}^{\mathrm{L}, \mathrm{R}}$ are the superconnections given by

$$
\Gamma_{\alpha}^{\mathrm{L}}=e^{-2 g V_{\mathrm{L}}} D_{\alpha} e^{2 g V_{\mathrm{L}}}, \quad \Gamma_{\alpha}^{\mathrm{R}}=e^{2 g V_{\mathrm{R}}} D_{\alpha} e^{-2 g V_{\mathrm{R}}} .
$$

Here $g$ is the gauge coupling constant. Then the higher-derivative interaction becomes

$$
\frac{1}{16} \int d^{4} \theta \Lambda\left(M, M^{\dagger}, V_{\mathrm{L}}, V_{\mathrm{R}}\right) \operatorname{Tr}\left[\overline{\mathcal{D}}_{\dot{\alpha}} M^{\dagger} e^{2 g V_{\mathrm{L}}} \mathcal{D}_{\alpha} M e^{-2 g V_{\mathrm{R}}} \overline{\mathcal{D}}^{\dot{\alpha}} M^{\dagger} e^{2 g V_{\mathrm{L}}} \mathcal{D}^{\alpha} M e^{-2 g V_{\mathrm{R}}}\right] .
$$

This term is invariant under the following $\mathrm{SU}(N)_{\mathrm{L}} \times \mathrm{SU}(N)_{\mathrm{R}}$ gauge transformations

$$
e^{2 g V_{\mathrm{L}, \mathrm{R}}} \longrightarrow e^{-i \lambda_{\mathrm{L}, \mathrm{R}}^{\dagger}} e^{2 g V_{\mathrm{L}, \mathrm{R}}} e^{i \lambda_{\mathrm{L}, \mathrm{R}}}, \quad M \longrightarrow e^{-i \lambda_{\mathrm{L}}} M e^{i \lambda_{\mathrm{R}}}, \quad M^{\dagger} \longrightarrow e^{-i \lambda_{\mathrm{R}}^{\dagger}} M^{\dagger} e^{i \lambda_{\mathrm{L}}^{\dagger}},
$$


for a gauge invariant real function $\Lambda\left(M, M^{\dagger}, V_{\mathrm{L}}, V_{\mathrm{R}}\right)$. Here $\lambda_{\mathrm{L}, \mathrm{R}}$ are the $\mathrm{SU}(N)_{\mathrm{L}} \times \mathrm{SU}(N)_{\mathrm{R}}$ chiral superfield gauge parameters. It is straightforward to show that the bosonic component from eq. (4.3) is given by

$$
\begin{aligned}
\Lambda\left(M, M^{\dagger}, A_{\mu}^{\mathrm{L}, \mathrm{R}}\right) \operatorname{Tr}[ & D_{\mu} M^{\dagger} D_{\nu} M D^{\mu} M^{\dagger} D^{\nu} M+\left(F^{\dagger} F\right)^{2}-D_{\mu} M^{\dagger} D^{\mu} M F^{\dagger} F \\
& \left.-D_{\mu} M D^{\mu} M^{\dagger} F F^{\dagger}\right]
\end{aligned}
$$

where $D_{\mu}$ is the gauge covariant derivative. The term (4.5) possesses the same structure found in the ungauged case. Therefore, we can trace the same procedure discussed in section 3 to find the solution of the auxiliary field $F$. The action for the $N=2$ case is the same as the one we found in section 3 , with all the derivatives in the interactions replaced by the gauge covariant derivative. We can also introduce the ordinary gauge kinetic term in the Lagrangian which would be relevant for the gauged BPS Skyrme model [61]. The equation of motion for the auxiliary field $D$ in the vector multiplet remains linear. We therefore integrate it out in a trivial way.

\section{Conclusion and discussion}

In this paper we have studied an $\mathcal{N}=1$ supersymmetric generalization of the Skyrme term in four spacetime dimensions. The model is based on the off-shell formulation of supersymmetric higher-derivative theories free from the auxiliary field problem. In order to find the Skyrme term, we consider an $\operatorname{SL}(N, \mathbb{C})$-valued chiral superfield $M$. Compared with the single-component case, the equation of motion for the auxiliary field, although algebraic, takes the form of a matrix equation and hence it is not straightforward to eliminate the auxiliary fields. We have, however, shown that the equation can be explicitly solved for the $N=2$ case, which is already a nontrivial case. When the superpotential is absent, the trivial solution $F=0$ is allowed. For this solution, the Lagrangian on the canonical branch does not admit the Skyrme term. For the non-canonical branch, we have also found that the on-shell Lagrangian generically depends on the double trace terms in addition to the single trace terms. If we restrict the $\mathrm{SL}(2, \mathbb{C})$-valued field $M$ to $M \in \mathrm{SU}(2)$, then the double-trace terms cancel out and leave behind only single trace terms; the resulting Lagrangian is then nothing but the Skyrme term. The situation is essentially the same even when we introduce the ordinary kinetic term $\int d^{4} \theta \operatorname{Tr}\left[M^{\dagger} M\right]$. We have found that the ordinary kinetic term $M_{\mu}^{\dagger} M^{\mu}$ cancels out on the non-canonical branch just as in the singlecomponent case. The Lagrangian on the non-canonical branch also admits the potential term (if we spoil the $G$-invariance of the Skyrme term) which originates from the Kähler tensor $\Lambda$.

We note that it is possible to introduce a superpotential into the model. In general, when one introduces a superpotential $W$, the equation of motion for the auxiliary field does not allow $F=0$ solution. For single-component models, analytic solutions to the auxiliary field equation have been found [34]. The superpotential induces quite non-linear higherderivative interactions in the on-shell action. It would be interesting to find solutions for 
the auxiliary field in our model with an added superpotential and study the structure of the higher-derivative interactions induced by $W$. Another interesting direction would be to study the supersymmetric completion of our model with eight supercharges.

Another important issue is to study configurations that keep fractions of supersymmetry in our model. One of the characteristic features of the off-shell higher-derivative supersymmetric models is its good accessibility to BPS equations. The BPS equations are obtained by the condition that the supersymmetry transformation of the fermions vanish. The equations are completely determined by the solution of the auxiliary field [34, 36]. Therefore, our result is quite important in the venue of finding BPS equations for Skyrmelike models. Finding soliton solutions in our model requires further investigations. We will come back to these issues in future studies.

The obvious generalization of our solution for the auxiliary field to $N>2$ could also be interesting. $N=3$ would require some work, but is probably doable. It would be interesting to see if a solution for arbitrary large $N$ can be found.

Finally, it would be interesting to study a manifestly supersymmetric version of the BPS Skyrme model, namely a model with a sixth-order derivative term.

\section{Acknowledgments}

S. B. G. thanks Roberto Auzzi for discussions. M. N. thanks Koji Hashimoto for discussions at the early stage of this work. S. B. G. also thanks the Recruitment Program of High-end Foreign Experts for support. The work of M. N. is supported in part by a Grant-in-Aid for Scientific Research on Innovative Areas "Topological Materials Science" (KAKENHI Grant No. 15H05855) and "Nuclear Matter in Neutron Stars Investigated by Experiments and Astronomical Observations" (KAKENHI Grant No. 15H00841) from the the Ministry of Education, Culture, Sports, Science (MEXT) of Japan. The work of M. N. is also supported in part by the Japan Society for the Promotion of Science (JSPS) Grant-in-Aid for Scientific Research (KAKENHI Grant No. 25400268) and by the MEXT-Supported Program for the Strategic Research Foundation at Private Universities "Topological Science" (Grant No. S1511006). The work of S. S. is supported in part by Kitasato University Research Grant for Young Researchers.

Open Access. This article is distributed under the terms of the Creative Commons Attribution License (CC-BY 4.0), which permits any use, distribution and reproduction in any medium, provided the original author(s) and source are credited.

\section{References}

[1] T.H.R. Skyrme, A Unified Field Theory of Mesons and Baryons, Nucl. Phys. 31 (1962) 556 [INSPIRE].

[2] T.H.R. Skyrme, A Nonlinear field theory, Proc. Roy. Soc. Lond. A 260 (1961) 127 [inSPIRE].

[3] E. Witten, Global Aspects of Current Algebra, Nucl. Phys. B 223 (1983) 422 [inSPIRE].

[4] E. Witten, Current Algebra, Baryons and Quark Confinement, Nucl. Phys. B 223 (1983) 433 [INSPIRE]. 
[5] R.A. Battye and P.M. Sutcliffe, Symmetric skyrmions, Phys. Rev. Lett. 79 (1997) 363 [hep-th/9702089] [INSPIRE].

[6] C.J. Houghton, N.S. Manton and P.M. Sutcliffe, Rational maps, monopoles and Skyrmions, Nucl. Phys. B 510 (1998) 507 [hep-th/9705151] [INSPIRE].

[7] R. Battye, N.S. Manton and P. Sutcliffe, Skyrmions and the alpha-particle model of nuclei, Proc. Roy. Soc. Lond. A 463 (2007) 261 [hep-th/0605284] [InSPIRE].

[8] P.H.C. Lau and N.S. Manton, States of Carbon-12 in the Skyrme Model, Phys. Rev. Lett. 113 (2014) 232503 [arXiv: 1408.6680] [InSPIRE].

[9] C.J. Halcrow and N.S. Manton, A Skyrme model approach to the spin-orbit force, JHEP 01 (2015) 016 [arXiv:1410.0880] [INSPIRE].

[10] M. Karliner, C. King and N.S. Manton, Electron Scattering Intensities and Patterson Functions of Skyrmions, arXiv:1510.00280 [INSPIRE].

[11] M. Haberichter, P.H.C. Lau and N.S. Manton, Electromagnetic Transition Strengths for Light Nuclei in the Skyrme model, arXiv:1510.08811 [INSPIRE].

[12] L.D. Faddeev, Some Comments on the Many Dimensional Solitons, Lett. Math. Phys. 1 (1976) 289 [INSPIRE].

[13] N.S. Manton and P.J. Ruback, Skyrmions in Flat Space and Curved Space, Phys. Lett. B 181 (1986) 137 [INSPIRE].

[14] C. Adam, J. Sanchez-Guillen and A. Wereszczynski, A Skyrme-type proposal for baryonic matter, Phys. Lett. B 691 (2010) 105 [arXiv: 1001.4544] [INSPIRE].

[15] C. Adam, J. Sanchez-Guillen and A. Wereszczynski, A BPS Skyrme model and baryons at large $-N_{c}$, Phys. Rev. D 82 (2010) 085015 [arXiv: 1007.1567] [InSPIRE].

[16] C. Adam, C. Naya, J. Sanchez-Guillen, R. Vazquez and A. Wereszczynski, The Skyrme model in the BPS limit, arXiv:1511.05160 [INSPIRE].

[17] E.B. Bogomolny, Stability of Classical Solutions, Sov. J. Nucl. Phys. 24 (1976) 449 [Yad. Fiz. 24 (1976) 861] [INSPIRE].

[18] E.A. Bergshoeff, R.I. Nepomechie and H.J. Schnitzer, Supersymmetric Skyrmions in Four-dimensions, Nucl. Phys. B 249 (1985) 93 [InSPIRE].

[19] B. Zumino, Supersymmetry and Kähler Manifolds, Phys. Lett. B 87 (1979) 203 [InSPIRE].

[20] L. Freyhult, The Supersymmetric extension of the Faddeev model, Nucl. Phys. B 681 (2004) 65 [hep-th/0310261] [INSPIRE].

[21] L.D. Faddeev and A.J. Niemi, Knots and particles, Nature 387 (1997) 58 [hep-th/9610193] [INSPIRE].

[22] J.M. Queiruga, Skyrme-like models and supersymmetry in 3+1 dimensions, Phys. Rev. D 92 (2015) 105012 [arXiv:1508.06692] [INSPIRE].

[23] J.M. Queiruga, private communication.

[24] A. Karlhede, U. Lindström, M. Roček and G. Theodoridis, Supersymmetric Nonlinear Maxwell Theories and the String Effective Action, Nucl. Phys. B 294 (1987) 498 [InSPIRE].

[25] I.L. Buchbinder, S. Kuzenko and Z. Yarevskaya, Supersymmetric effective potential: Superfield approach, Nucl. Phys. B 411 (1994) 665 [InSPIRE]. 
[26] I.L. Buchbinder, S.M. Kuzenko and A. Yu. Petrov, Superfield chiral effective potential, Phys. Lett. B 321 (1994) 372 [INSPIRE].

[27] S.J. Gates Jr., Why auxiliary fields matter: The Strange case of the 4-D, $N=1$ supersymmetric QCD effective action, Phys. Lett. B 365 (1996) 132 [hep-th/9508153] [INSPIRE].

[28] S.J. Gates Jr., Why auxiliary fields matter: The strange case of the 4-D, $N=1$ supersymmetric QCD effective action. 2., Nucl. Phys. B 485 (1997) 145 [hep-th/9606109] [INSPIRE].

[29] M. Nitta, A Note on supersymmetric WZW term in four dimensions, Mod. Phys. Lett. A 15 (2000) 2327 [hep-th/0101166] [INSPIRE].

[30] C. Adam, J.M. Queiruga, J. Sanchez-Guillen and A. Wereszczynski, N=1 supersymmetric extension of the baby Skyrme model, Phys. Rev. D 84 (2011) 025008 [arXiv:1105.1168] [INSPIRE].

[31] C. Adam, J.M. Queiruga, J. Sanchez-Guillen and A. Wereszczynski, BPS bounds in supersymmetric extensions of K field theories, Phys. Rev. D 86 (2012) 105009 [arXiv:1209.6060] [INSPIRE].

[32] J. Khoury, J.-L. Lehners and B. Ovrut, Supersymmetric $P(X, \phi)$ and the Ghost Condensate, Phys. Rev. D 83 (2011) 125031 [arXiv:1012.3748] [InSPIRE].

[33] C. Adam, J.M. Queiruga, J. Sanchez-Guillen and A. Wereszczynski, Extended Supersymmetry and BPS solutions in baby Skyrme models, JHEP 05 (2013) 108 [arXiv: 1304.0774] [INSPIRE].

[34] M. Nitta and S. Sasaki, BPS States in Supersymmetric Chiral Models with Higher Derivative Terms, Phys. Rev. D 90 (2014) 105001 [arXiv:1406.7647] [INSPIRE].

[35] M. Nitta and S. Sasaki, Higher Derivative Corrections to Manifestly Supersymmetric Nonlinear Realizations, Phys. Rev. D 90 (2014) 105002 [arXiv: 1408.4210] [INSPIRE].

[36] M. Nitta and S. Sasaki, Classifying BPS States in Supersymmetric Gauge Theories Coupled to Higher Derivative Chiral Models, Phys. Rev. D 91 (2015) 125025 [arXiv:1504.08123] [INSPIRE].

[37] S. Bolognesi and W. Zakrzewski, Baby Skyrme Model, Near-BPS Approximations and Supersymmetric Extensions, Phys. Rev. D 91 (2015) 045034 [arXiv:1407.3140] [InSPIRE].

[38] S.R. Coleman, J. Wess and B. Zumino, Structure of phenomenological Lagrangians. 1., Phys. Rev. 177 (1969) 2239 [INSPIRE].

[39] C.G. Callan Jr., S.R. Coleman, J. Wess and B. Zumino, Structure of phenomenological Lagrangians. 2., Phys. Rev. 177 (1969) 2247 [INSPIRE].

[40] M. Bando, T. Kuramoto, T. Maskawa and S. Uehara, Structure of Nonlinear Realization in Supersymmetric Theories, Phys. Lett. B 138 (1984) 94 [INSPIRE].

[41] M. Bando, T. Kuramoto, T. Maskawa and S. Uehara, Nonlinear Realization in Supersymmetric Theories, Prog. Theor. Phys. 72 (1984) 313 [InSPIRE].

[42] M. Bando, T. Kuramoto, T. Maskawa and S. Uehara, Nonlinear Realization in Supersymmetric Theories. 2., Prog. Theor. Phys. 72 (1984) 1207 [INSPIRE].

[43] K. Itoh, T. Kugo and H. Kunitomo, Supersymmetric Nonlinear Realization for Arbitrary Kählerian Coset Space G/H, Nucl. Phys. B 263 (1986) 295 [INSPIRE]. 
[44] K. Itoh, T. Kugo and H. Kunitomo, Supersymmetric Nonlinear Lagrangians of Kählerian Coset Spaces G/H: G=E6, E7 and E8, Prog. Theor. Phys. 75 (1986) 386 [inSPIRE].

[45] W. Lerche, On Goldstone Fields in Supersymmetric Theories, Nucl. Phys. B 238 (1984) 582 [INSPIRE].

[46] G.M. Shore, Supersymmetric Higgs Mechanism With Nondoubled Goldstone Bosons, Nucl. Phys. B 248 (1984) 123 [INSPIRE].

[47] A.C.W. Kotcheff and G.M. Shore, Kähler $\sigma$ Models From Supersymmetric Gauge Theories, Int. J. Mod. Phys. A 4 (1989) 4391 [inSPIRE].

[48] J. Wess and J. Bagger, Supersymmetry and supergravity, Princeton University Press, Princeton U.S.A. (1992), pg. 1-259.

[49] S. Sasaki, M. Yamaguchi and D. Yokoyama, Supersymmetric DBI inflation, Phys. Lett. B 718 (2012) 1 [arXiv:1205.1353] [INSPIRE].

[50] M. Roček and A.A. Tseytlin, Partial breaking of global D $=4$ supersymmetry, constrained superfields and three-brane actions, Phys. Rev. D 59 (1999) 106001 [hep-th/9811232] [INSPIRE].

[51] I. Antoniadis, E. Dudas and D.M. Ghilencea, Supersymmetric Models with Higher Dimensional Operators, JHEP 03 (2008) 045 [arXiv:0708.0383] [INSPIRE].

[52] M. Gomes, J.R. Nascimento, A. Yu. Petrov and A.J. da Silva, On the effective potential in higher-derivative superfield theories, Phys. Lett. B 682 (2009) 229 [arXiv:0908.0900] [INSPIRE].

[53] S.M. Kuzenko and S.J. Tyler, The one-loop effective potential of the Wess-Zumino model revisited, JHEP 09 (2014) 135 [arXiv:1407.5270] [INSPIRE].

[54] G.M. Shore, Geometry of Supersymmetric $\sigma$ Models, Nucl. Phys. B 320 (1989) 202 [INSPIRE].

[55] G.M. Shore, Geometry of Supersymmetric $\sigma$ Models. 2. Fermions, Connections and Currents, Nucl. Phys. B 334 (1990) 172 [InSPIRE].

[56] K. Higashijima, M. Nitta, K. Ohta and N. Ohta, Low-energy theorems in $N=1$ supersymmetric theory, Prog. Theor. Phys. 98 (1997) 1165 [hep-th/9706219] [InSPIRE].

[57] M. Nitta, Moduli space of global symmetry in $N=1$ supersymmetric theories and the quasiNambu-Goldstone bosons, Int. J. Mod. Phys. A 14 (1999) 2397 [hep-th/9805038] [INSPIRE].

[58] M. Ostrogradsky, Memoires sur les equations differentielles relatives au probleme des isoperimetres, Mem. Ac. St. Petersbourg VI 4 (1850) 385.

[59] M. Koehn, J.L. Lehners and B.A. Ovrut, Higher-Derivative Chiral Superfield Actions Coupled to N=1 Supergravity, Phys. Rev. D 86 (2012) 085019 [arXiv:1207.3798] [InSPIRE].

[60] F. Farakos and A. Kehagias, Emerging Potentials in Higher-Derivative Gauged Chiral Models Coupled to $N=1$ Supergravity, JHEP 11 (2012) 077 [arXiv:1207.4767] [INSPIRE].

[61] C. Adam, C. Naya, J. Sanchez-Guillen and A. Wereszczynski, The gauged BPS baby Skyrme model, Phys. Rev. D 86 (2012) 045010 [arXiv: 1205.1532] [INSPIRE]. 